Pacific Journal of Mathematics

CERTAIN REPRESENTATIONS OF INFINITE GROUP ALG ERas 


\section{CERTAIN REPRESENTATIONS OF INFINITE GROUP ALGEBRAS}

\section{SinHA}

For any group $G$, let $\rho$ be an irreducible representation of the group algebra $\mathfrak{\preccurlyeq} G$ over a field $\mathfrak{F}$. Then by Schur's lemma, the center $\Delta$ of its commuting ring, is a field con-

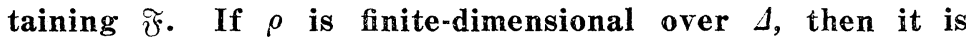
called finite and if it is finite-dimensional over $\widetilde{F}$ itself, then it is called strongly finite. In this paper, certain conditions are given for finiteness of $\rho$. Also it is shown that for some types of groups, finiteness of $\rho$ is related to the existence of abelian subgroups of finite index in certain quotient of the group. Conditions under which finiteness and strongly finiteness are equivalent, are given. Finally, consequences of $\rho$ being faithful on $G$, or being faithful on $\widetilde{F}$, are studied.

Study of finiteness of irreducible representations was initiated by Kaplansky in [3], and later carried to a great extent by Passman, Issacs, and others: \{see [5] and relevent references therein\}. Finiteness and strong finiteness were studied in [6]. Using a slight modification of the technique of [4] to suit our nonsemisimple case, we get Theorem 1 which includes the results of [4] and gives us Theorem 2 whose corollaries contains the result of [3].

We further recall the well-known result that for a finite group $G$, if the kernel of an irreducible representation $o$ contains the commutator subgroup $G^{\prime}$, then the representation is 1-dimensional over 4. As corollary to our Theorem 3, we prove that in general, if $G^{\prime}$ is contained in the kernel of $\rho$, then $\rho$ is finite whether $G$ is finite or not.

2. Finiteness of representation. In this section we study conditions under which a given irreducible representation is finite, and also the conditions under which all irreducible representations are finite. We need the following:

Definitions. 1. Let $\rho$ be a representation of $\mathfrak{F} G$. Then $G_{0}=$ $\{g \in G \mid \rho(g)=1\}$, and Kern $\rho=$ kernel $\rho=\{x \in \mathfrak{F} G \mid \rho(x)=0\}$.

Thus $\rho$ is $G$-faithful if $G_{\rho}=1$, while $\rho$ is $\mathfrak{F} G$-faithful if Kern $\rho=0$.

2. Let $\mathfrak{B} \leqq$ Aut $G$. For $S \leqq G$, we shall write $\mathfrak{V}_{\mathscr{B}}(S)$ for the left-ideal $\left\{\sum x_{i}\left(\mathscr{S}_{i}^{\beta_{i}}-1\right) \mid x_{i} \in \mathfrak{F} G, \mathscr{S}_{i} \in S, \beta_{i} \in \mathfrak{B}\right\}$. \{For a general study of such ideals we may refer to [6] and [8].\} We write $\mathfrak{I}(S)$, if $\mathfrak{B}=\{$ identity $\}$. 
3. We also define the $\mathfrak{B}$-kernel of $\rho$ in $H \leqq G$ to be

$$
\left\{h \in H \mid \rho\left(h^{\beta}\right)=1, \forall \beta \in \mathfrak{B}\right\},
$$

and set $K_{n}^{\mathfrak{g}}(H)=\bigcap\{\mathfrak{B}$-kernels of $\rho$ in $H\}$, where the intersection runs through all irreducible representations $\rho$ of $G$ for which $\operatorname{dim}_{\Delta} \rho>n^{2}$, where $\Delta$ is the center of the commuting ring of $\rho$. If no such $\rho$ exists, then we put $K_{n}^{\mathfrak{g}}(H)=G$.

4. Let $S_{2 n}$ be the symmetric group of degree $2 n$. Then for an algebra $A$, the sums

$$
\sigma_{n}=\sum_{\sigma \in S_{2 n}}(\operatorname{sgn} \sigma) x_{\sigma(1)} \cdot x_{\sigma(2)} \cdots x_{\sigma(2 n)}, x_{\rho} \in A,
$$

are called the Standard Monomial Sums (of parity $n$ ).

5. Define $\sum_{n}(G)$ to be the $\widetilde{F}$-space spanned by all the $\sigma_{n}$ in $\mathfrak{F} G$. \{We shall frequently write $\sum_{n}$ wherever the group in question is clear from context.\}

This $\sum_{n}$ plays a significant role in determining the degrees of irreducible representations.

Specifically we have:

Proposition 1. Let $\rho$ be an irreducible representation of $\mathfrak{F} G$. Then $\operatorname{dim}_{\Delta} \rho \leqq n^{2}$ if and only if $\sum_{n} \leqq \operatorname{Kern} \rho$.

Proof. If $\operatorname{dim}_{\Delta} \rho \leqq n^{2}$ then $\mathfrak{F} G / \operatorname{Kern} \rho$ is a primitive algebra of matrices of $\operatorname{dim} n$ over 4 . By Theorem 1 of [1], for any $\sigma_{n} \in \mathfrak{F} G$, $\rho\left(\sigma_{n}\right)=0$, whence $\sigma_{n} \in \operatorname{Kern} \rho$ so that $\Sigma_{n} \subseteq \operatorname{Kern} \rho$.

Conversely, suppose $\sum_{n} \subseteq \operatorname{Kern} \rho$. Then $\mathfrak{\mho} G / \operatorname{Kern} \rho$ is a primitive algebra satisfying $\sigma_{n}=0$ for every $\sigma_{n}$ in $\mathfrak{F} G / \operatorname{Kern} \rho$. Then by Theorem 1 of [2], p. 226, $\Im G / \operatorname{Kern} \rho$ is a central simple algebra of $\operatorname{dim} \leqq n^{2}$. Hence $\operatorname{dim}_{4} \rho \leqq n^{2}$.

Using this result we obtain:

THEOREM 1. Let $S \leqq H \leqq G$. Then $S \subseteq K_{n}^{\text {g }}(H)$ if and only if $\mathfrak{A}_{\mathfrak{B}}(S) \cdot \sum_{n} \subseteq \operatorname{Rad} \mathfrak{\mho} G$.

Proof. We observe that $\mathfrak{N}_{\mathfrak{B}}(S)=\left\{\sum x_{i}\left(s_{i}^{\beta_{i}}-1\right) \mid x_{i} \in \mathfrak{\mho} G, s_{i} \in S, \beta_{i} \in \mathfrak{B}\right\}$. Thus, to show that $\mathfrak{U}_{\mathfrak{B}}(S) \cdot \sum_{n} \subseteq \operatorname{Rad} \mathfrak{F} G$, it suffices to show that $\left(s^{\beta}-1\right) \cdot \sum_{n} \subseteq \operatorname{Rad} \mathfrak{F} G$, for $\forall \beta \in \mathfrak{B}, \forall s \in S$. Now let $h \in K_{n}^{\mathfrak{g}}(H)$ and $\rho$ be an irreducible representation of $G$. If $\operatorname{dim}_{\Delta} \rho>n^{2}$, then $\rho\left(h^{\beta}\right)=1$ or $\rho\left(h^{\beta}-1\right)=0, \forall \beta \in \mathfrak{B}$, by the very difinition of $K_{n}^{\mathfrak{B}}(H)$. On the other hand, if $\operatorname{dim}_{\Delta} \rho \leqq n^{2}$, then by Proposition $1, \rho\left(\sum_{n}\right)=0$. 
Thus, in both cases, $\rho\left[\left(h^{\beta}-1\right) \cdot \sum_{n}\right]=0$. Since $\rho$ is arbitrary, so $\left(h^{\beta}-1\right) \cdot \sum_{n} \subseteq \operatorname{Rad} \mathfrak{F} G$. Hence $S \subseteq K_{n}^{\mathfrak{g}}(H)$ implies $\mathfrak{U}_{\mathfrak{B}}(S) \cdot \sum_{n} \subseteq$ $\operatorname{Rad} \mathfrak{F} G$.

Conversely, suppose $\mathfrak{V}_{\mathfrak{B}}(S) \cdot \sum_{n} \leqq \operatorname{Rad} \mathfrak{F} G$. Then, in particular,

$$
\left(s^{\beta}-1\right) \cdot \sum_{n} \subseteq \operatorname{Rad} \mathfrak{\Im} G, \forall \beta \in \mathfrak{B}, s \in S .
$$

We define the left-idealisor [7], of $\sum_{n}$ into $\operatorname{Rad} \mathfrak{\Im} G$, by $L_{\mathrm{Rad}}\left(\sum_{n}\right)_{\Im G}=$ $L\left(\sum_{n}\right)=\left\{x \in \mathfrak{F} G \mid x \cdot \sum_{n} \subseteq \operatorname{Rad} \mathfrak{F} G\right\}$. This is clearly a left-ideal. Also $\left[L\left(\sum_{n}\right) \cdot g\right] \cdot \sum_{n}=L\left(\sum_{n}\right)\left[g \cdot \sum_{n} \cdot g^{-1}\right] \cdot g=\left[L\left(\sum_{n}\right) \cdot \sum_{n}\right] \cdot g \subset \operatorname{Rad} \mathfrak{F} G \cdot g=$ $\operatorname{Rad} \mathfrak{\mho} G$, for $\forall g \in G$. Hence $L\left(\sum_{n}\right)$ is a two-sided ideal of $\mathfrak{F} G$.

Now let $\rho$ be an irreducible representation of $G$, afforded by the $\mathfrak{F} G$-module $\mathfrak{M}$. Since $L\left(\sum_{n}\right)$ is a two-sided ideal, so Ann $L\left(\sum_{n}\right)=$ $\left\{m \in \mathfrak{M} \mid L\left(\sum_{n}\right) m=0\right\}$ is an $\mathfrak{F} G$-submodule of $\mathfrak{M}$. Thus either Ann $L\left(\sum_{n}\right)=0$ or $\mathfrak{M}$. Now assume that $\operatorname{dim}_{\Delta} \rho>n^{2}$. Again, by Proposition 1, $\rho\left(\sum_{n}\right) \neq 0$ so that $\sum_{n} \cdot \mathfrak{M} \neq 0$, whence $\sum_{n} \nsubseteq \operatorname{Rad} \mathfrak{F} G$. But $L\left(\sum_{n}\right) \cdot\left[\sum_{n} \cdot \mathfrak{M}\right]=\left[L\left(\sum_{n}\right) \cdot \sum_{n}\right] \cdot \mathfrak{M}=0$, since $L\left(\sum_{n}\right) \cdot \sum_{n} \subseteq \operatorname{Rad} \mathfrak{F} G$. Thus Ann $L\left(\sum_{n}\right)=\mathfrak{M}$. Then, as by hypothesis $s^{\beta}-1 \in L\left(\sum_{n}\right)$, so $\left(s^{\beta}-1\right) \cdot \mathfrak{d}=0$; or $\rho\left(s^{\beta}-1\right)=0$. As $\rho$ was arbitrary with $\operatorname{dim}_{\lrcorner} \rho>$ $n^{2}$; so $s \in K_{n}^{2}(H)$.

Letting $\widetilde{\mho}=$ complex-field, we have $\operatorname{Rad} \mathfrak{\Im} G=0$. Taking $\mathfrak{B}=\{1\}$ in this case, we obtain the result of Passman [4]:

Corollary. $g \in K_{n}(G)$ if and only if $(g-1) \cdot \Sigma_{n}=0$.

We also deduce:

THEOREM 2. Let $S \leqq G$ and $\mathfrak{B} \leqq$ Aug $G$ such that $S^{\Re}=G$. Then $\mathfrak{U}_{\mathscr{s}}(S) \cdot \sum_{n} \leqq \operatorname{Rad} \mathfrak{F} G$ if and only if $\operatorname{dim}_{\Delta} \rho \leqq n^{2}$ for every irreducible representation $\rho$ of $G$. $\{$ Of course, $\Delta$ depends on $\rho$.

Proof. By Theorem $1, \mathfrak{2}_{\mathfrak{y}}(S) \cdot \sum_{n} \subseteq \operatorname{Rad} \mathfrak{F} G$ if and only if $S \subseteq$ $K_{n}^{\mathfrak{B}}(G):\{G=H\}$.

The latter condition is equivalent to the statement that for every irreducible representation $\rho$ with $\operatorname{dim}_{\Delta} \rho>n^{2}$, we have $\rho\left(s^{\beta}\right)=1$, $\forall \beta \in \mathfrak{B}, s \in S$. Since $S^{\Re}=G$, so we deduce that $\rho=1$.

COROLLARY 1. If $\sum_{n} \subseteq \operatorname{Rad} \mathfrak{\Im} G$ for some $n$, then every irreducible representation of $\mathfrak{F} G$ is finite.

Corollary 2. [3]. If in $\mathfrak{F} G, \sum_{n}=0$ for some $n$, then every irreducible representation of $G$ is finite.

Next recall that if $|G|<\infty$ then $G^{\prime} \subseteq G_{\rho}$ for any irreducible 
representation $\rho$, if and only if $\rho$ is of $\operatorname{dim}$. 1. A generalization of sort, is obtained in the corollary to:

THEOREM 3. Let $\rho$ be an irreducible representation of $\Im G$.

(a) If either (i) $\sum_{n}\left(G / G_{\rho}\right)=0$ for some $n$, or (ii) $\exists A \leqq G \ni$. $G_{\rho} \leqq A,|G: A|<\infty$ and $A / G_{\rho}$ is abelian, then $\rho$ is finite.

(b) (Conversely) If $\rho$ is finite and $\mathfrak{F} G$ satisfies either of the following conditions:

(i) $G / G_{\rho}$ is periodic and $\mathfrak{\mho}\left(G / G_{\rho}\right)$ is nonmodular;

(ii) $G / G_{\rho}$ is periodic with a finite p-Sylow subgroup for Char. $\mathfrak{F}=p \neq 0$;

(iii) $G / G_{\rho}$ satisfies minimum-condition on subgroups; then $\exists A \leqq G \ni \cdot G_{\rho} \leqq A,|G: A|<\infty$ and $A / G_{\rho}$ is abelian.

Proof. (a) Suppose (i) holds. In the notation of [6], $G_{\rho}=$ $\mathfrak{U}^{-1}(\operatorname{Kern} \rho)$ where for any ideal $I$ of $\mathfrak{\mho} G, \mathfrak{A}^{-1}(I)=\{g \in G \mid g-1 \in I\}$, and hence $\mathfrak{A}\left(G_{\rho}\right)$ is a sub-ideal in Kern $\rho$. Since $\mathfrak{X}\left(G_{\rho}\right)$ is the kernel of the linear extension of the cannonical map $G \rightarrow G / G_{\rho}$, so $\mathfrak{F} G / \mathfrak{I}\left(G_{\rho}\right) \cong$ $\mathfrak{F}\left(G / G_{\rho}\right)$. Therefore, $\sum_{n}\left(G / G_{\rho}\right)=0$ implies that the standard monomialsum, in $\mathfrak{F} G / \mathfrak{A}\left(G_{\rho}\right)$, all vanish. Now $\mathfrak{F} G / \operatorname{Kern} \rho \cong \mathfrak{F} G / \mathfrak{A}\left(G_{\rho}\right) /$ Kern $\rho / \mathfrak{A}\left(G_{\rho}\right)$; therefore, the same holds for $\mathfrak{F} G / \operatorname{Kern} \rho$. In particular, $\sum_{n}(G) \cong \operatorname{Kern} \rho$. Then, by Proposition $1, \rho$ is finite. Next let (ii) hold. Then $\left|G / G_{\rho}: A / G_{\rho}\right|=n<\infty$, and $A / G_{\rho}$ is abelian. Therefore, by the result of Kaplansky mentioned before, or by Theorems. 5.1, 8.1 of [5], all the irreducible representations of $G / G_{\rho}$ are finite.

Now if $\rho$ is afforded by the $\widetilde{\Im} G$-module $\mathfrak{M}$, then putting $\bar{\rho}(\bar{g}) \cdot m=$ $\rho(g) \cdot m$, for $\bar{g} \in G / G_{\rho}$, and observing that $G_{\rho}=\{g \in G \mid \rho(g)=1\}$, we get a representation $\bar{\rho}$ of $G / G_{\rho}$, such that $\bar{\rho}$ is irreducible and the commuting ring of $\bar{\rho}$ in $\mathrm{Hon}_{\mathfrak{s}}(\mathfrak{M}, \mathfrak{M})$, is the same as that of $\rho$.

Thus the finiteness of $\bar{\rho}$-implies the finiteness of $\rho$.

(b) $G / G_{\rho} \cong S \leqq G L(n, \Delta)$ and any such $S$ satisfying either of the conditions (i), (ii) or (iii), is abelian by finite: \{see [9], Corollaries 9.4, 9.7, 9.8, and 9.23\}. We then get our $A$, by taking the complete inverse-image of the abelian part of $G / G_{\rho}$.

Since the group-algebra of an abelian group always satisfies $\Sigma_{n}=0$, so we obtain:

CoRollary. If $G^{\prime} \leqq G_{\rho}$, then $\rho$ is finite.

3. Strong finiteness of representations. In this section we give a result which shows the equivalence of finiteness and strongfiniteness in certain conditions. 
THEOREM 4. Under either of the following conditions, an irreducible representation $\rho$ of $G$ is finite if and only if it is strongly finite:

(i) $G$ is finitely generated;

(ii) $\rho$ is absolutely irreducible;

(iii) $\exists H \unlhd G \ni|G: H|<\infty$ and $\rho_{H}$ has a strongly finite constituent.

Proof. (i) This is the content of Lemma 7 of [6].

(ii) Let the absolutely irreducible finite representation $\rho$, be afforded by the $\mathfrak{F} G$-module $\mathfrak{M}$. Since $\Delta \leqq \mathrm{Hom}_{\mathfrak{F}}(\mathfrak{M}, \mathfrak{M})$, $\mathfrak{F} \leqq \Delta$, so we can make $\Delta \boldsymbol{\bigotimes}_{\widetilde{s}} \mathfrak{M}$ into a $\Delta G$-module by letting $g \cdot(d \otimes m)=$ $d \otimes \rho(g) m$.

Define $\psi: \Delta \bigotimes_{\mathfrak{s}} \mathfrak{M} \rightarrow \mathfrak{M}$ by $\psi(d \otimes m)=d m$. Since $\rho$ and $d$ commute, so

$$
\psi(g \cdot(d \otimes m))=\psi(d \otimes \rho(g) m)=d(\rho(g) m)=\rho(g)(d m) .
$$

Thus $\psi$ is a $\Delta G$-homomorphism. Since $\mathfrak{M}$ is absolutely irreducible, so $\Delta \bigotimes_{\widetilde{r}} \mathfrak{M}$ is irreducible. So $\psi$ is an isomorphism. Then $\operatorname{dim}_{\Delta}\left(\Delta \bigotimes_{\widetilde{r}} \mathfrak{M}\right)=$ $\operatorname{dim}_{\Delta} \mathfrak{M}<\infty$. Thus $\rho$ is also finite-dimensional over $\mathfrak{F}$.

(iii) By Clifford's theorem, $\rho_{H}=\bigoplus \sum_{i=1}^{[G ; H]} \rho_{i}$, where $\rho_{i}$ are all conjugate irreducible-representations of $H$. Hence, if one of them is finite-dimensional over $\widetilde{F}$, then so are all; and hence $\rho$.

4. Faithful representation. Finally, let $\rho$ be a representation (not necessarily irreducible) of the group algebra $\mathfrak{F} G$. For any leftideal $I$ we shall write $\rho^{I}$ for the representation afforded by the module $I \cdot \mathfrak{M}$, where $\rho$ is afforded by $\mathfrak{M}$. We shall let $\mathfrak{X}=\mathfrak{X}(G)$ denote the augmentation-ideal of $\mathfrak{F} G$ and $J=[\mathfrak{F} G, \mathfrak{F} G]$. Let Char $\widetilde{\gamma}=p \neq 0$.

We then investigate the consequences of $\rho$ being faithful as a representation of $G$ and as a representation of $\mathfrak{F} G$ respectively. Recalling that if $H \leqq G$, then $\mathfrak{U}(H)$ is the left-ideal in $\mathfrak{F} G$ generated by $\{h-1 \mid h \in H\}:[6]$, we have the following:

Theorem 5. (a) If $\rho$ is faithful on $G$, then $A=\mathfrak{Z}^{-1}\left(\operatorname{Kern} \rho^{\text {q }}\right)$ is an elementary abelian normal p-subgroup which is central if $\mathfrak{A} \subseteq \operatorname{Kern} \rho^{\mathfrak{x}}$.

(b) If $\rho$ is faithful on $\mathfrak{\mho} G$, then

(i) $A=1$ unless $|G|=2, p=2$ in which case $A=G$;

(ii) $B=\mathfrak{U}^{-1}\left(\operatorname{Kern} \rho^{J}\right)=1$ unless $p=2$ and $B=G$ is abelian, or $p=2, G$ is nonabelian and $B$ is central.

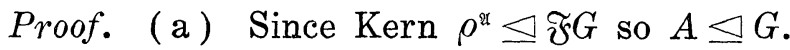


Let $\mathfrak{M}$ afford $\rho$ so that $\mathfrak{A} \cdot \mathfrak{M}$ affords $\rho^{\mathfrak{x}}$. Hence $g \in A$ if and only if $(g-1) \cdot \sum_{x \in G} \lambda_{x}(x-1) m=0$ for each $m \in \mathfrak{M}$. Since $p \neq 0$, so $\left(g^{p}-1\right) m=(g-1)^{p} m=(g-1)\left[(g-1)^{p-1} m\right]=0$ as $(g-1)^{p-1} m \in$ $\mathfrak{A} \cdot \mathfrak{M}$. Thus $g^{p} m=m$ and faithfulness implies that $g^{p}=1$. Further, if $h \in A$ then $(h-1) m$ and $(g-1) m$ are both in $\mathfrak{A} \cdot \mathfrak{M}$. Then,

$$
(g-1)(h-1) m=0=(h-1)(g-1) m
$$

or

$$
g^{-1} h^{-1} g h m=m \text {. }
$$

Again faithfulness gives that $g h=h g$; i.e., $A$ is abelian.

If $\mathfrak{U} \subseteq$ Kern $\rho^{2}$, then $g \in G, h \in A$ implies

$$
\begin{aligned}
(g h-1) m & =[(g-1)(h-1)+(g-1)+(h-1)] m \\
& =(g-1) m+(h-1) m=(h g-1) m .
\end{aligned}
$$

Thus $g h m=h g m$ and faithfulness gives that $A \subseteq Z(G)$.

(b) (i) Now let $\rho$ be $\Im G$-faithful. Then the Kern $\rho^{\text {q }}=$ Ann $\mathfrak{U}$ in $\mathfrak{F} G$. It is well-known that this annihilator is 0 unless $|G|<\infty$ and Ann $\mathfrak{A}=\mathfrak{F} \cdot\left(\sum_{g \in G} g\right)$. Now if $g_{i} \in A$, then $g_{i}-1=$ $a \in \operatorname{Kern} \rho^{\text {I }}$ so that $g_{i}-1=k \cdot \sum g, k \in \mathfrak{F}$. Linear independence of the group elements, gives us that $g_{i}=1$, and $k=0$, or $|G|=2$, $g_{1}=1, i=2, k=1$, and $+1=-1$ in $\mathfrak{F}$.

(ii) Again by faithfulness $\operatorname{Kern} \rho^{J}=\operatorname{Ann} J$. So $g \in B$ implies that $(g-1)(h k-k h)=0, \forall h, k \in G$, i.e., $g h k-g k h-h k+k h=0$. If Char, $\widetilde{F} \neq 2$, then we must either have $g h k=g k h$ in which case $h k=k h$, or $g h k=h k$ in which case $g=1$.

In case Char $\widetilde{F}=2$ and $g$ is noncentral then choose $k \in G$ such that $g k \neq k g$. Put $h=g^{-1}$. Then the above identity gives,

$$
k-g k g^{-1}-g^{-1} k+k g^{-1}=0 \text {. }
$$

Since $g k g^{-1} \neq k$, so either $k=g^{-1} k$ or $k=k g^{-1}$, both leading to $g=1$, a contradiction. Thus in this case $g \in Z(G)$.

\section{REFERENCES}

1. S. A. Amitsur and J. Levitzki, Minimal identities for algebras, Proc. Amer. Math. Soc., 1 (1950), 449-463.

2. N. Jacobson, Structure of Rings, Amer. Math. Soc. Coll. Publ., 28 1964, Rhode Island, Providence.

3. I. Kaplansky, Groups with representations of bounded degree, Canad. Math. J., 1 (1949), 575-580.

4. D. S. Passman, Character kernels in discrete groups, Proc. Amer. Math. Soc., 17 (1966), 487-492.

5 .

Infinite Group Rings, Marcel-Dekker Inc., N. Y., 1971. 
6. I. Sinha, On the augmentation maps of the subgroups of a group, Math. Zeitsch., 94 (196)6, 193-206.

7. I. Sinha and J. Srivastava, Ideals in commutative domains, Math. Mag., 44 (3), (1971), 124-127.

8. J. Srivastava, Ph. D. Thesis, I. I. T., Kanpur, India.

9. B. A. F. Wehrfritz, Infinite Linear Groups, Queen Mary College Math. Notes, 1969.

Received April 18, 1973 and in revised form October 16, 1973.

Michigan State University 



\section{PACIFIC JOURNAL OF MATHEMATICS}

\section{EDITORS}

RICHARD ARENS (Managing Editor)

University of California

Los Angeles, California 90024

R. A. Beaumont

University of Washington

Seattle, Washington 98105
J. DugundJI

Department of Mathematics University of Southern California Los Angeles, California 90007

D. Gilbarg AND J. Milgram Stanford University

Stanford, California 94305

\section{ASSOCIATE EDITORS}
E. F. BECKENBACH
B. H. NeUmanN
F. WOLF
K. YOSHIDA

\section{SUPPORTING INSTITUTIONS}

UNIVERSITY OF BRITISH COLUMBIA CALIFORNIA INSTITUTE OF TECHNOLOGY

UNIVERSITY OF CALIFORNIA

MONTANA STATE UNIVERSITY

UNIVERSITY OF NEVADA

NEW MEXICO STATE UNIVERSITY

OREGON STATE UNIVERSITY

UNIVERSITY OF OREGON

OSAKA UNIVERSITY
UNIVERSITY OF SOUTHERN CALIFOF

STANFORD UNIVERSITY

UNIVERSITY OF TOKYO

UNIVERSITY OF UTAH

WASHINGTON STATE UNIVERSITY UNIVERSITY OF WASHINGTON

AMERICAN MATHEMATICAL SOCIET NAVAL WEAPONS CENTER 


\section{Pacific Journal of Mathematics}

\section{Vol. 54, No. 1 \\ May, 1974}

Ralph K Amayo, Engel Lie rings with chain conditions ..................

Bernd Anger and Jörn Lembcke, Hahn-Banach type theorems for hypolinear

functionals on preordered topological vector spaces ..................

Gregory Frank Bachelis and Samuel Ebenstein, On $\Lambda(p)$ sets ................

Harvey Isaac Blau, Indecomposable modules for direct products of finite

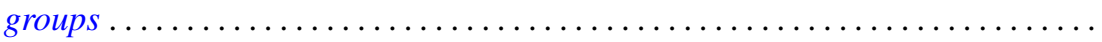

Larry Eugene Bobisud and James Calvert, Singular perturbation of a

time-dependent Cauchy problem in a Hilbert space ................

Walter D. Burgess and Robert Raphael, Abian's order relation and orthogonal

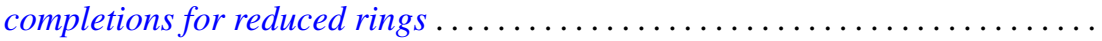

James Diederich, Representation of superharmonic functions mean continuous at

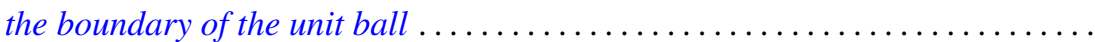

Aad Dijksma and Hendrik S. V. de Snoo, Self-adjoint extensions of symmetric

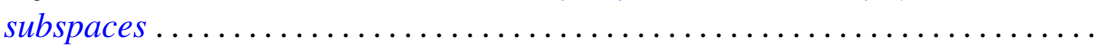

Gustave Adam Efroymson, A Nullstellensatz for Nash rings . . . . . . . . . . . . .

John D. Elwin and Donald R. Short, Branched immersions onto compact orientable surfaces . . . . . . . . . . . . . . . . . . . . . . . . .

John Douglas Faires, Comparison of the states of closed linear

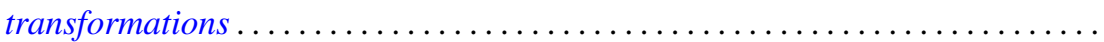

Joe Wayne Fisher and Robert L. Snider, On the von Neumann regularity of rings with regular prime factor rings .

Franklin Takashi Iha, A unified approach to boundary value problems on compact intervals

Palaniappan L. Kannappan and Che Tat $\mathrm{Ng}$, On functional equations connected with directed divergence, inaccuracy and generalized directed divergence

Samir A. Khabbaz and Elias Hanna Toubassi, The module structure of Ext $(F, T)$ over the endomorphism ring of $T$...

Garo K. Kiremidjian, On deformations of complex compact manifolds with boundary.

Dimitri Koutroufiotis, Mappings by parallel normals preserving principal

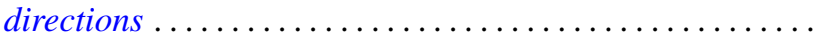

W. K. Nicholson, Semiperfect rings with abelian adjoint group

Norman R. Reilly, Extension of congruences and homomorphisms to translational hulls

Sadahiro Saeki, Symmetric maximal ideals in $M(G)$

Brian Kirkwood Schmidt, On the homotopy invariance of certain functors ...

H. J. Shyr and T. M. Viswanathan, On the radicals of lattice-ordered rings ...

Indranand Sinha, Certain representations of infinite group algebras ...

David Smallen, The group of self-equivalences of certain complexes ...

Kalathoor Varadarajan, On a certain problem of realization in homotopy

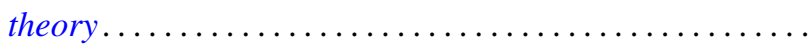

